\title{
Humour et émotivité : l'expression des sentiments dans Le Voyage de Charlemagne
}

\author{
Parody and Emotivity: Expression of Sentiments \\ in Charlemagne's Journey?
}

\author{
Alain Corbellari \\ Universités de Lausanne et de Neuchâtel \\ alain.corbellari@unil.ch
}

\begin{abstract}
We propose here to analyze from an emotional point of view Charlemagne's Journey, which is undoubtedly the funniest chanson de geste (except the atypical Audigier). In particular, we will try to see to what extent the irony of the text influences the representation of emotions, and how these influence in turn the reception of the text by the reader.
\end{abstract}

Keywords: chanson de geste, irony, emotions in litterature

Analyser les émotions dans les textes médiévaux n'est pas chose facile, car elles sont souvent exprimées à travers des expressions stéréotypées qui nous renseignent davantage sur un certain imaginaire général de l'émotivité que sur les cas particuliers que nous serions heureux de pouvoir cerner dans les œuvres littéraires.

Cependant, bien que ce soit dans la chanson de geste que les formules revêtent les formes les plus contraignantes, c'est sur un texte épique que j'aimerais ici me pencher, car il présente un aspect de distanciation qui le détache nettement du tout venant des chansons de geste. Parmi la centaine de textes épiques que nous a léguée le Moyen Âge français, s'il ne manque pas de récits qui mettent à mal les figures d'autorité, Le Voyage de Charlemagne à Jérusalem et à Constantinople est sans doute le seul ${ }^{1}$

\footnotetext{
${ }^{1} \mathrm{Si}$ l'on exclut le scatologique et tout à fait hors normes Audigier, dont l'appartenance thématique au genre épique pose d'ailleurs problème. Voir l'édition conjointe de ces deux textes que je viens de
} 
dans lequel le traitement des héros traditionnels nous fait douter du sérieux des intentions de l'auteur. Le sourire est certes présent dans nombre de chansons de geste, en particulier dans celles du cycle de Guillaume d'Orange (voir Corbellari, 2011), mais nulle part n'est véritablement remise en question l'exaltation héroïque des protagonistes. Dans Le Voyage de Charlemagne, en revanche, les motivations de l'empereur à la barbe fleurie n'apparaissent pas toujours totalement claires et sa conduite - ainsi que celle de ses douze pairs - déroge franchement au sérieux que l'on est en droit d'attendre de lui. Dans la mesure où ce texte déjoue beaucoup des stéréotypes de la chanson de geste, mais en conserve au moins tout autant, il m'a semblé intéressant de l'analyser dans la perspective de l'émotivité pour voir dans quelle mesure ses aspects incongrus pouvaient dépasser ou conforter les attentes traditionnelles du genre épique.

Rappelons-en, à gros traits, l'intrigue : vexé que sa femme lui ait dit qu'elle trouvait plus de prestance à l'empereur Hugon de Constantinople, Charlemagne décide d'aller se mesurer à ce monarque, et, pour donner un peu plus de dignité à son expédition, fait un crochet par Jérusalem où il acquiert une douzaine de précieuses reliques. Le passage à Constantinople n'en est pas moins le but réel du voyage, puisque les trois quarts de cette chanson extrêmement courte (elle ne comprend que 870 vers) sont consacrés à l'épisode byzantin : après une arrivée en fanfare, Charlemagne et les douze pairs se livrent, dans la chambre qui leur a été attribuée, à des rodomontades, les fameux gabs, qu'ils croient proférés à l'abri des oreilles indiscrètes; malheureusement, un espion les a entendus et rapporte tout à Hugon qui, très fâché, somme les Francs, sous peine de mort, d'accomplir les exploits qu'ils se sont vantés de pouvoir réaliser. Avec l'aide d'un ange, trois des gabs sont accomplis. Hugon décide alors d'arrêter les frais (car son empire est menacé d'être mis sens dessus dessous) et se déclare le vassal de Charlemagne.

Ce qui frappe d'emblée dans ce texte, c'est l'extrême susceptibilité des personnages. Certes, la chanson de geste, et la littérature médiévale en général, abonde en réactions violentes de ses héros, souvent fort chatouilleux sur les questions d'honneur ; mais ici tout semble à la fois exacerbé et, paradoxalement, mis à distance, tant les enjeux de la narration peuvent sembler futiles en regard des luttes à mort contre les païens et des inexpiables querelles féodales qui font habituellement le fond des chansons de geste. Or, dans la mesure où, justement, nous sommes dans un texte souriant et, somme toute, pacifique (Charlemagne et ses hommes ont entrepris leur expédition sans emporter la moindre arme offensive), les émotions qui s'y font jour ont des chances d'être, sinon plus authentiques, du moins plus originales que celles des épopées traditionnelles.

publier : L'Epopée pour rire (Le Voyage de Charlemagne à Jérusalem et à Constantinople et Audigier), 2017. C'est mon édition (et ma traduction) que je citerai ici. 
Le texte démarre d'emblée dans l'émotionnel, et nous allons voir que cette première scène contient in nuce tout le reste de la chanson. Charlemagne se livre à une cérémonie solennelle que l'on pourrait dire de « recouronnement » au monastère de Saint-Denis, et, alors qu'il a demandé à sa femme si elle connaissait un roi qui portait mieux sa couronne que lui, elle lui répond sans ménagements que c'est bien le cas. La réaction de Charlemagne ne se fait pas attendre :

Quant l'entend Charlë[un], mult [en] est corecez ;

Pur Franceis ki l'oïrent, mult [en] est enbrunchez. (Voyage, 2017, p. 128, v. 17-18)

(À ces mots, Charlemagne est fort contrarié ;

et comme ses Francs l'écoutent, il baisse la tête.)

À l'affront personnel s'ajoute la honte devant ses hommes. Les deux formules sont traditionnelles : le courroux associe, on le sait (comme son exact synonyme ire), le chagrin et la colère, et le chief embronc (la tête penchée) est l'attitude typique de la mélancolie et de l'abattement (voir Ménard, 1969). Mais Charlemagne se reprend bien vite, et, en véritable homme d'action, décide aussitôt d'aller voir ce roi, non sans menacer sa femme :

Se vus me avez mentid, vus le cumperez cher :

Trencherai vus la teste od me espee d'acer. (Voyage, 2017, p. 128, v. 24-25)

(mais si vous m'avez menti, vous le payerez cher :

je vous trancherai la tête de mon épée d'acier !)

La reine se repent alors d'avoir trop parlé :

De la plus haulte tur de Paris la citez

Me larrai cuntreval par creance devaler

Que pur la vostre hunte ne fud dit ne pensed. (Voyage, 2017, p. 130, v. 36-38)

(De la plus haute tour de la ville de Paris,

je me laisserai tomber pour prouver

que je ne l'ai ni dit ni pensé pour votre honte.)

Charlemagne la dissuade de commettre l'irréparable mais réitère, au vers 42 , sa menace de décapitation, motif peu courant, dans son caractère excessif, de l'écriture épique, et qui va parcourir tout Le Voyage de Charlemagne.

Lorsque le roi Hugon apprend que les Français ont ri de lui, il profère en effet la même menace : «Trancherai lur les testes od ma spee furbie ! ( « je leur trancherai la tête de mon épée effilée ! », Voyage, 2017, p. 178, v. 633).

Le vers sera répété presque à l'identique, un peu plus bas, devant les hommes de Charlemagne rassemblés (« Trancherai vus les testes od ma spee furbie », Voyage, 2017 , p. 180, v. 647). Et un peu plus loin encore, à l'intention d'Olivier, qui a parié 
qu'il ferait cent fois l'amour en une nuit à la fille d'Hugon, s'il échouait dans son pari («Trancherai lui la teste a ma spee furbie », Voyage, 2017, p. 184, v. 698).

Situation paradoxale : plutôt que de s'émouvoir du déshonneur de sa fille, Hugon, aveuglé par sa colère, menace de mort Olivier, pour le cas où celui-ci manquerait à sa parole ! Certains critiques se sont émus de ce manque de sensibilité paternelle ${ }^{2}$, mais derrière cet apparent « irréalisme » il y a une observation émotionnelle (ne parlons pas ici de «psychologie »!) plus fine qu'il n'y paraît : le narrateur, par la récurrence mécanique de la formule liée à la décapitation, nous dresse le portrait d'un personnage chez qui un sens exacerbé de l'honneur a étouffé toute tendresse et toute attention à l'autre. Remarquons, cela dit, qu'Olivier lui-même avait proposé spontanément en proférant son $g a b$ devant ses amis, d'encourir le même sort en cas de défaillance : «Demain perde la teste par covent le otrai » (« je serai d'accord de me faire couper la tête le lendemain », Voyage, 2017, p. 166, v. 489).

L'obsession d'Hugon (qui prend la suite de celle que Charlemagne manifestait au début de la chanson pour le même geste), réapparaît encore à propos de Guillaume, deuxième guerrier à tenter d'avérer son $g a b$ : «Trancherai lui la teste a mun brant acerin » (Voyage, 2017, p. 186, v. 742).

Mais Hugon se gardera de menacer le troisième guerrier, Bernard (il utilisera en fait une autre formule que nous évoquerons plus loin), et une fois le troisième $g a b$ réussi, deviendra doux comme un agneau; en une scène qui fait écho à celle qui ouvrait la chanson, il accepte de porter sa couronne aux côtés de Charlemagne, mais «plus bas » que ce dernier :

Karlemaines portet la grant corone a or,

Li reis Hugues la sue plus basement un poi. (Voyage, 2017, p. 192, v. 809-10)

(Charlemagne porte sa couronne d'or,

Le roi Hugues la sienne, mais un peu plus bas.)

Comme s'il avait été lui-même décapité !

D'une expression presque triviale, signalant l'affectivité immédiate des personnages, l'auteur a donc fait un motif dont la symbolique est parfaitement transparente : tout l'enjeu du texte est de présenter des personnages désireux d'avoir « une tête de plus » que les autres, en recourant au besoin, ou en menaçant de recourir, à une manière drastique de faire disparaître les têtes qui dépassent! De Charlemagne à sa femme et au roi Hugon, en passant par tous les pairs, dont les rodomontades ne servent au fond qu'à les grandir au détriment des autres, on assiste ainsi au déploie-

\footnotetext{
${ }^{2}$ Le plus véhément a sans doute été Paul Aebischer (1956) qui traite Olivier de « juponnier sans vergogne », de « grossier et facile vainqueur », de « dernier des truands » de «plus amoral des lovelaces » et même de « saligaud », et Hugon, de père indigne « qui prostitue sa fille pour avoir le plaisir de couper le cou à ses hôtes ».
} 
ment de ce que René Girard appelle la « violence mimétique » qui fait de chacun le rival de tous (voir Girard, 1972). Bien plus qu'un artifice rhétorique, la menace de décapitation exemplifie - à travers une formule émotionnelle brute qui est un véritable cri du cœur - cette volonté de suprématie, qui est finalement dévolue sans partage au seul Charlemagne.

Mais cette figure de l'abaissement en rejoint une autre: celle de la chute. L'épouse de Charlemagne proposait, on l'a vu, de se précipiter du haut d'une tour élevée pour racheter sa faute. Ce faisant, elle imaginait combler le désir de son mari de la voir rabaissée plus bas que terre. Mais l'empereur des Francs n'envisageait pas une solution aussi extrême, car avoir sa femme à ses côtés lui permet justement de faire valoir par contraste sa propre grandeur. Et c'est exactement pour la même raison qu'il tiendra, à la fin du texte, à voir Hugon parader à ses côtés : l'essentiel est que la différence de taille soit affichée.

Auparavant, pourtant, Charlemagne aura lui-même fait son « expérience de la tour ». Arrivé à Constantinople, il est en effet convié par Hugon à contempler, au sommet de la tour de son palais, des automates qui font souffler les vents à volonté, dans le but, justement, de faire se mouvoir l'édifice. Mais il s'agit en fait d'un piège ; si Hugon est habitué à ce mouvement giratoire, Charlemagne et ses hommes, en revanche, ne le sont pas et se retrouvent complètement étourdis par l'expérience, ce que le narrateur ne manque pas de souligner en évoquant une forte réaction affective des Français :

Franceis sunt tuz verset, ne se poent tenir

E cov(e)rirent lur chef e adenz e suvin,

E dist li uns a l'altre : «Mal sumes entrepris ;

Les portes sunt uvertes, si n'en poüm issir. » (Voyage, 2017, p. 158, v. 388-91)

(Les Français ont tous été renversés, ils ne peuvent résister

et couvrent leurs têtes par devant et par derrière.

Les uns disent aux autres : « Pauvres de nous,

les portes sont ouvertes et pourtant nous ne pouvons pas sortir. »)

Ils n'est ainsi pas interdit de voir les gabs comme une revanche sur l'humiliation subie ici par les guerriers carolingiens. Mais une autre vengeance, plus précise, leur sera offerte au moment de la réalisation du troisième gab; celui-ci consiste en effet en une inondation dont Hugon ne peut se protéger qu'en montant, seul, au sommet de sa tour, tandis que les Francs se réfugient sur un vieux pin :

En la plus halte tur li reis s'en fuid a ped.

Desur un pin antif est Carles al vis fer,

Il e li duze pers, li barun chevaler,

[E] prient Dampnedeu qui des eauls ait pited. (Voyage, 2017, p. 190, v. 779-82) 
(le roi s'enfuit à toutes jambes dans la plus haute tour.

Charles au visage farouche se tient sur un vieux pin,

lui et les douze pairs, ses nobles chevaliers ;

ils prient le Seigneur Dieu qu'il ait pitié d'eux.)

Notons que, bien qu'ils soient eux-mêmes responsables de l'inondation - qu'ils devraient donc maitriser - les hommes de Charlemagne sont tout de même pris d'une réaction de crainte qui les pousse à demander la protection divine. À l'insensibilité d'Hugon, ils répondent donc par une soumission au Créateur qui accentue, par contraste, leur humilité ; je reviendrai sur ce motif.

Par ailleurs, le pin sur lequel monte Charlemagne (alors qu'il est au contraire généralement installé sous un pin !) entre en isotopie ${ }^{3}$ avec un autre arbre de même espèce évoqué quelques vers plus haut, au moment où Hugon rêve encore de pouvoir se débarrasser des faiseurs de paris stupides :

Mais si un [sul] en fault, par Deu omnipotent,

Demain les f[e]rai pendre ensum cel pin al vent,

A unes forz estaches n'en av(e)runt raidement. (Voyage, 2017, p. 188, v. 759-61)

(mais si un seul y manque, par le Dieu tout-puissant,

je les ferai pendre au sommet de ce pin demain en plein vent,

à une potence solide, et il n'y aura pour eux nulle rémission.)

On a passé de la décapitation à la pendaison, mais l'élévation dont rêve Hugon a fini par tourner à sa confusion: Charlemagne et ses hommes se retrouvent bien dans un pin, mais, loin que celui-ci soit l'instrument de leur perte, il les accueille pour leur salut.

On rappellera que tout arbre, dans la littérature médiévale, et dans la chanson de geste en particulier, est susceptible de désigner la transcendance (voir Labbé, 1987). Ainsi, un pin, lui aussi antif (vers 594), était déjà évoqué par Bertrand dans son gab, où il pariait qu'il parviendrait, sous cet arbre justement, à mettre en pièces trois solides boucliers. Dans La Chanson de Roland, c'est également sous un pin que meurt Roland, dont l'âme va être emportée au Paradis. Curieusement, pourtant, au début du Voyage de Charlemagne ce n'est pas sous un pin mais sous un olivier que l'empereur parade avec son épouse! À l'idée d'une influence méridionale sur le récit, on préférera voir un clin d'œil à l'un des principaux guerriers de Charlemagne $^{4}$, dont le gab mènera d'ailleurs à la conception d'un héros, Galien le restoré, qui, dans les versions tardives de la légende de Roland, sera le vengeur des guerriers

\footnotetext{
${ }^{3}$ Il convient donc de préférer le mot pin à la lecture pui, « colline », proposée par Madeleine Tyssens dans sa traduction critique du Voyage de Charlemagne à Jérusalem et à Constantinople (1978), substantif qui s'accorde de toute façon mal avec l'adjectif antif.

${ }^{4}$ On est ainsi tenté de lire dans ce parallèle un sous-entendu grivois : l'autre femme du récit, la fille de l'empereur, ne se retrouve-t-elle pas elle aussi sous un... Olivier !?
} 
morts à Roncevaux. Apparemment éloignées de la question de l'émotivité, ces quelques remarques sur les arbres nous permettent ainsi de souligner les qualités affectives dont peuvent être porteurs les éléments naturels dans les textes médiévaux.

Si l'on revient plus précisément à l'intrigue du Voyage de Charlemagne, on soulignera maintenant qu'à l'ensemble des occurrences que nous avons relevées jusqu'ici d'une affectivité virile exprimant avec violence la rivalité mimétique s'opposent les manifestations d'une l'affectivité pieuse, dont le texte n'est pas avare. La scène du don des reliques à Charlemagne par le patriarche de Jérusalem occupe en effet une place centrale et même fondatrice à l'intérieur du cycle épique carolingien ${ }^{5}$. De fait, il se pourrait qu'en dépit des traces manifestes de parodie dans de nombreux épisodes de la chanson, la scène des reliques véhicule plus de sérieux que l'ont dit nombre de commentateurs. Certes, certaines de ces reliques peuvent de prime abord paraître bizarres, voire grotesques (du lait de la Vierge, des poils de la barbe de saint Pierre...) mais toutes sont attestées et entourées, au Moyen Âge, de la plus grande vénération ${ }^{6}$. La scène qui préside à la rencontre de Charlemagne et du patriarche ne peut cependant manquer de faire sourire : aussitôt arrivé à Jérusalem, l'empereur à la barbe fleurie s'est en effet assis avec un parfait sans-gêne à la Table de la Cène, en compagnie de ses douze pairs ; un Juif qui passait par là, croyant voir le Christ revenu avec ses disciples, court annoncer au patriarche que ce spectacle l'a décidé à se convertir. Intrigué, le patriarche se rend alors à la vénérable église et fait la connaissance de l'illustre voyageur, que sa réputation avait précédé, et dont il reconnaît aussitôt la grandeur. Pas de rivalité mimétique, ici, mais des assauts de politesse entre l'empereur, qui se voit officiellement décerner le titre de «Charlemagne », et le patriarche :

E dist li patriarches : « Sire, mult estes beer!

Sis as en la chaëre u sist m[eïs]mes Deus.

Aiés nun Charle[maine]s sur tuz reis curunez. »

E dist li empereres : « Cin cenz merciz de Deu !

De voz saintes reliques si vus plaist me donez,

Que porterai en France qu'en voil enluminer. » (Voyage, 2017, p. 138-140, v. 156-61)

(Le patriarche dit alors : «Seigneur, quelle bravoure est la vôtre !

Asseyez-vous donc sur le siège où Dieu lui-même s'assit

et que le nom de Charlemagne soit élevé au-dessus de ceux de tous les rois couronnés. »

L'empereur lui répond : «Soyez-en, par Dieu, remercié cinq cents fois !

Donnez-moi s'il vous plaît une part de vos saintes reliques,

que je rapporterai en France, pour en illustrer mon royaume. »)

\footnotetext{
${ }^{5}$ Le Voyage de Charlemagne est ainsi en quelque sorte le premier volet d'un petit « cycle des reliques » dans l'épopée médiévale. Voir Le Person (2013).

${ }^{6}$ Voir le long développement que leur consacre Carla Rossi dans son édition du texte (Il Viaggio, 2006).
} 
Le vers « Karlemaines l'en rent saluz e amistez » ( Charlemagne lui témoigne sa reconnaissance par des saluts et des protestations d'amitié. ») revient ensuite à trois reprises en moins de trente vers (v. 166, 182, 190, la troisième avec inversion de «amisté » et «saluz » pour les besoins de l'assonance). Et c'est un véritable potlatch qui s'esquisse au moment où Charlemagne doit faire ses adieux à la ville sainte, tant lui et le patriarche rivalisent de générosité :

« Faites .C. mulz receivere d'or e d'argent trusset. »

E dist li patriarches : « Ja ma[r] en parlerez !

Tuz li mens granz tresors vus seit abandunez;

Tant en prengent Franceis cum en vuldrent porter. » (Voyage, 2017, p. 144, v. 220-23)

(«Veuillez recevoir ces cent mulets chargés d'or et d'argent. »

Le patriarche dit alors : «Ne redites jamais cela!

Que tous mes grands trésors vous soient abandonnés ;

Que les Français en prennent autant qu'ils peuvent en emporter. »)

Ce que Charlemagne ne conquerra que de haute lutte à Constantinople, à savoir la soumission de son homologue, qui s'exprimera d'ailleurs en des vers semblables (« Trestuz mes granz tresor vus sei[e]nt abandunez; / Tant en prengent Franceis cum il en voldrent porter ! - « Que tous mes grands trésors vous soient abandonnés ; / que les Français en prennent tout ce qu'ils voudront emporter ! », Voyage, 2017, p. 194-196, v. 839-40), il l'obtient spontanément du patriarche de Jérusalem. Les formules, témoignant d'une émotivité positive et pleine de déférence, sont ici le signe d'une entente parfaite, d'un raffinement de politesse qui nous change de la rudesse habituelle des héros épiques. In fine, Charlemagne pourra également échanger des marques d'affectivité avec l'empereur Hugon (« Vunt sei entrebaiser, a Deu sunt cumandez » - «Ils s'embrassent mutuellement et se recommandent à Dieu », Voyage, 2017, p. 196, v. 848), mais le «salut et amitié » reste l'apanage de sa relation au patriarche.

On comprend mieux ici le sens de l'intervention angélique dans la réalisation des gabs, et la confiance sereine que Charlemagne et ses pairs manifesteront envers Dieu lors de l'inondation de Constantinople. Au sein d'un corpus épique où la confiance en Dieu est souvent exprimée de manière froidement formulaire et où de nombreux textes manifestent même une certaine forme d' " oubli de Dieu » ${ }^{7}$, Le Voyage de Charlemagne se signale comme l'un des plus franchement religieux ; le sujet, bien sûr, y prédispose, mais son traitement manifeste bien le désir de son

\footnotetext{
${ }^{7}$ C'est évidemment le cas de nombre de « barons révoltés » dont l'obstination revêt souvent un caractère quelque peu diabolique (Raoul de Cambrai en reste l'un des exemples les plus emblématiques) ; mais même un héros aussi positif que Guillaume d'Orange se signale par la rareté de ses appels à Dieu.
} 
auteur d'illustrer un type de spiritualité privilégiant une relation directe et personnelle avec Dieu.

Que nous apprend, en fin de compte, le rapide survol des marques d'émotion qui ponctuent, dans Le Voyage de Charlemagne, les relations de l'empereur d'Occident avec ses rivaux potentiels ou réels (je n'excepte pas Dieu lui-même de cette catégorie) ? Tout d'abord que, sur ce chapitre du moins, rien n'y signale à coup sûr la tonalité parodique que l'on se plaît à retrouver dans le déroulement de maint épisode de la chanson. L'hyperbole est autant sinon plus le privilège du genre épique que la marque distinctive d'un texte particulier et n'est jamais à elle seule la marque d'une intentionnalité comique. La brièveté du récit donne, certes, l'impression, qu'il est saturé de marques affectives, et ce fait contribue sans nul doute à la vivacité de sa narration et à l'allure humoristique qu'il revêt ${ }^{8}$. Mais ces marques pourraient bien être au service d'un projet plus sérieux que la simple dérision. Rendant palpable la tension de ses relations à son épouse puis à Hugon, elle mettent, par ailleurs, d'autant mieux en évidence la sorte d'immédiate complicité qui lie Charlemagne au patriarche de Jérusalem - et par celui-ci à Dieu lui-même - ce qui permet à la fois de faire oublier que les relations avec Rome (qui avaient des chances d'apparaître plus conflictuelles) sont complètement évacuées de la chanson, et de dessiner entre Charlemagne et la source du christianisme primitif le lien le plus direct qui soit. L'affection que se manifestent Charles et le patriarche ne sont ainsi pas sans évoquer l'agapè des premiers chrétiens et si le voyage à Constantinople fera resurgir les conflits qui minent l'exercice conjoint du pouvoir temporel, le lien spirituel est en revanche subsumé par une concorde idéale, celle justement qui se concrétisera dans l'efficacité immédiate conférée par les reliques aux gabs des guerriers carolingiens. L'intervention de l'ange, y compris dans la réalisation du peu convenable pari d'Olivier (même si, dans ce cas précis, elle n'est pas explicitement mentionnée, revêt sans doute un aspect ironique, mais cette protection, en même temps, confirme trop bien l'axiologie politique et morale du récit pour ne pas être totalement mise sur le compte de la dérision.

Charlemagne n'aura jamais été aussi affectivement proche des autres protagonistes de ses aventures. Mais c'est que l'enjeu et de taille : l'amour, la dévotion et la rivalité se livrent ici un rude combat dont le grand vainqueur sera l'empereur d'Occident, monarque attachant car attaché, et toujours prêt, dans le meilleur comme dans le pire, à faire prévaloir ses sentiments sur sa raison : si les gabs n'ont pas le sens commun, c'est aussi parce que leur rôle est moins d'illustrer la pure bravoure

\footnotetext{
${ }^{8} \mathrm{Ne}$ donnons qu'un seul exemple : au vers 71, Charlemagne donne pour prétexte à sa décision de partir en Orient, le fait qu'il en a fait à trois reprise le rêve. Comme ces songes ne sont pas décrits, on a la forte impression qu'ils n'ont existé que dans l'imagination de l'empereur et qu'ils n'ont ainsi que de prétexte fallacieux à la motivation de ses hommes. Cependant, ces rêves sont bel et bien décrits dans les sources latines du Voyage de Charlemagne. L'équivalence brièveté = humour, qui semble s'imposer à première lecture, n'est donc pas sûre !
} 
que la folie du risque que prend Charlemagne pour imposer sa propre gloire. Tout finit bien, car la générosité, chez Charles, l'emporte sur le soupçon. Même si les relations exactes de sa femme au roi Hugon, ne seront jamais totalement éclaircies", il sait qu'il ne coupera la tête à personne et que sa jalousie n'existe que pour le plaisir de la dépasser dans l'amour chrétien.

\footnotetext{
${ }^{9}$ Dans un récit celtique tardif, mais puisant peut-être aux mêmes sources que Le Voyage de Charlemagne, l'Histoire du Roi Arthur et du Roi Cornwall, la femme à la parole imprudente est l'ancienne épouse de celui dont elle fait l'éloge intempestif à son nouveau mari.
} 


\section{BIBLIOGRAPHIE}

Aebischer, P. (1956). Le gab d'Olivier. Revue belge de philologie et d'histoire, 34 (3), 659-79.

Corbellari, A. (2011). Guillaume d'Orange ou la naissance du héros médiéval. Paris : Klincksieck. Girard, R. (1972). La Violence et le sacré. Paris : Grasset.

Il Viaggio di Carlo Magno a Gerusalemme e a Costantinopoli (2006). C. Rossi (Ed., Trans.). Alessandria : Edizioni dell'Orso.

Labbé, A. (1987). L'Architecture des palais et des jardins dans les chansons de geste. Essai sur le thème du roi en majesté. Paris - Genève : Champion - Slatkine.

Le Person, M. (2013). Le pouvoir merveilleux, surnaturel et sacré des reliques de la Passion dans le «Petit cycle des reliques » («Le Voyage de Charlemagne à Jérusalem et à Constantinople, La Destruction de Rome et Fierabras »). In M. Possamaï-Perez, J.-R. Valette (Eds.), Chanter de geste. L'Art épique et son rayonnement. Hommage à Jean-Claude Vallecalle (pp. 221-240). Paris : Champion.

Le Voyage de Charlemagne à Jérusalem et à Constantinople (1978). M. Tyssens (Trans.). Gand : Story-Scientia.

Le Voyage de Charlemagne à Jérusalem et à Constantinople (2017). A. Corbellari (Ed., Trans.). In A. Corbellari (Ed.), L'Épopée pour rire (Le Voyage de Charlemagne à Jérusalem et à Constantinople et Audigier). Paris : Champion.

Ménard, Ph. (1969). « Tenir le chief embronc, crosler le chief, tenir la main a la maissele » : trois attitudes de l'ennui dans les chansons de geste du XII ${ }^{\mathrm{e}}$ siècle. In Société Rencesvals, IV congrès international (Heidelberg 28 août-2 septembre 1967), Actes et Mémoires (pp. 145-155). Heidelberg : Carl Winter. 